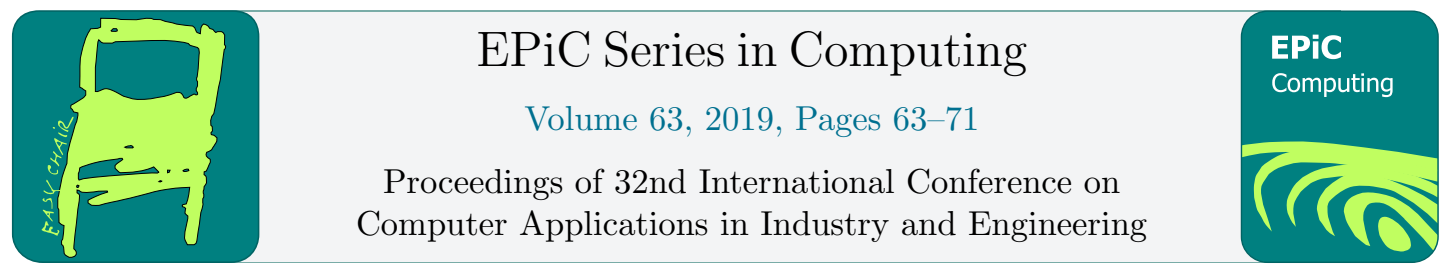

\title{
A biometric for shark dorsal fins based on boundary descriptor matching
}

\author{
Taina Coleman and Jucheol Moon \\ Department of Computer Engineering and Computer Science \\ California State University Long Beach \\ Long Beach, CA, 90840, USA \\ tainagdcoleman@gmail.com \\ jucheol.moon@csulb.edu
}

\begin{abstract}
Recent progress in animal biometrics has revolutionized wildlife research. Cutting edge techniques allow researchers to track individuals through noninvasive methods of recognition that are not only more reliable, but also applicable to large, hard-to-find, and otherwise difficult to observe animals. In this research, we propose a metric for boundary descriptors based on bipartite perfect matching applied in shark dorsal fins. In order to identify a shark, we first take a fin contour and transform it to a normalized coordinate system so that we can analyze images of sharks regardless of orientation and scale. Finally, we propose a metric scheme that performs a minimum weight perfect matching in a bipartite graph. The experimental results show that our metric is applicable to identify and track individuals from visual data.
\end{abstract}

keywords: Biometric for sharks, Shark dosal fin, Boundary descriptor, Bipartite graph, Minimum weight perfect matching

\section{Introduction}

The ability to recognize individuals is a requirement for several aspects of ecological research and essential for environmental conservation [16]. Research in animal identification started at the end of 1960's and first results were presented in 1976 [19]. Biometrics is a science usually applied to humans, but it has showed to be very promising for identifying, classifying, tracking, monitoring diseases in animal population [1]. Since the beginning, main studies were developed around cattle for their economy importance in agriculture and the fairly easy access to the animals, but it has been expanding to other species as technology evolves.

In the past, the technology existent to identify animals was generally invasive like ear tags, leg rings, collars, number tattoos and hot branding. Not only some of these procedures could potentially hurt the animals, they were also not reliable since tags and collars could just fall off, resulting in financial and data loss. Advances in computer vision combined with unique animal characteristics provided non-invasive solutions to animal identification [10, 2]. Initially, these

Q. Yuan, Y. Shi, L. Miller, G. Lee, G. Hu and T. Goto (eds.), CAINE 2019 (EPiC Series in Computing, vol. 63), pp. 63-71 
systems were strongly reliable on large sets of unconstrained data, preferably taken in natural conditions in order to facilitate the identification work done manually or semi-manually [21, 9, $24,18,22]$.

The next step was to research fully automated systems capable to locate, identify and classify data. Such discoveries would then be the bridge for more advanced animal recognition and biometrics systems like the ones recently available. This approach eliminates the subjective human observer iteration and allows biologists to collect and process data in a scale not reachable before. Generally, this methods require a combination of a framework to detect the animal against a noise background and a biometric system to identify the unique features and match them [10]. A recent example of a fully automated identification system is the deep learning framework for cattle recognition. Based on the uniqueness and immutable nature of cattle's muzzles beads and ridges, it was possible to develop a system that applies point image pattern to identify specific individuals [11].

A different approach emerged to satisfy the need of biometric identification that was not dependent on texture. Hughes and Burghardt [8] introduced the idea is based on an already existent technique of using the fin shape to track sharks manually and semi-automatically [23], and its focus is on the contour information of smooth or textureless objects, specifically applied to great white shark dorsal fins in an unconstrained environment. This was the first partially automated contour-based visual ID system in animal biometrics [8].

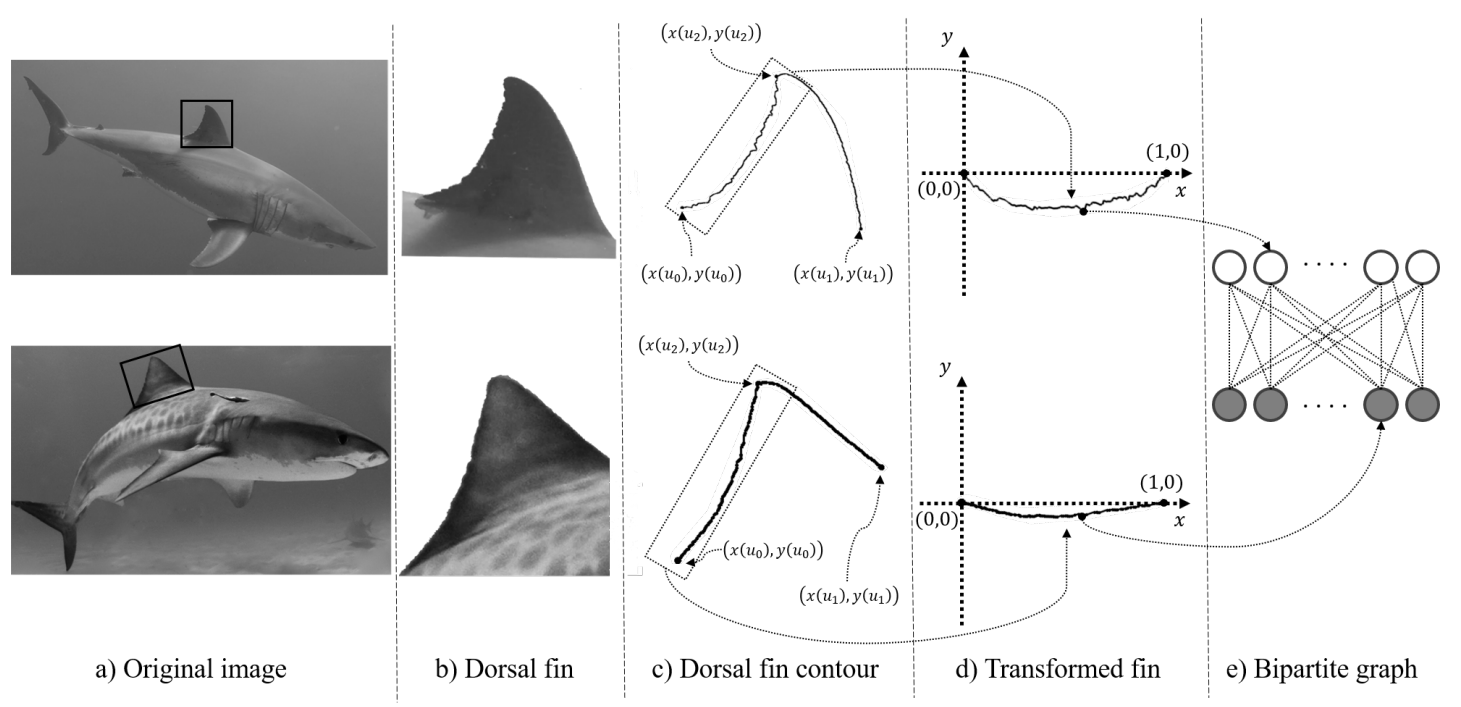

Figure 1: Overview of the metric design

The intention of this paper is to propose a novel biometric design for boundary descriptors based on bipartite perfect matching, and apply it into shark identification, seeking to later use it in a big data setting to track an individual shark. We utilize the work of extracting dorsal fins, then, inspired by Affine Transformations as presented in [5], the contour of the extracted dorsal fin is translated to a normalized coordinate system in order to guarantee orientation and scale invariance. Such properties are required on the use of our bipartite perfect matching based metric.

Minimum weight perfect matching in a bipartite graph has already been shown successful in designing a metric $[14,17]$. Inspired by these previous works, we designed a metric that can 
be computed in three steps. First, given two sets of dorsal fin contour keypoints, we create a bipartite graph such that every keypoint in the first set shares an edge with every key point in the second set. This is a fully-connected bipartite graph. Then, we weight each edge by calculating the Euclidean Distance between each of the nodes. Lastly, we perform a minimum weight perfect matching algorithm [4], and the sum of the edge's weights of the perfect matching is our metric.

\section{Related Work}

Animal biometrics are defined by the measurable data related to appearance aspects [12], movement characteristics and vocalizations of animals that can be used as biometric entities [6]. In order to be a suitable biometric entity, such traits must be measurable by a recording device, adequately permanent, characteristic of the animal class of interest and universally displayed throughout the relevant population, such as the stripes on zebras [13] and tigers [7] or the spots on cheetahs [9]. However, not every physical characteristic fits the definition, for example, the injury marks in white sharks are salient and unique, but they heal quickly and such temporal variability can limit their use as biometric entity for long-term studies [10, 3].

Recently, deep learning approaches like in [11] are very popular. Their goal was to develop a successful non-invasive biometrics framework to manage cattle. The work involved preparing a database of muzzle point images, extracting the salient texture features and representation of muzzle point image using convolution neural network and deep belief neural network, and the use of a denoising auto-encoder technique to encode the extracted features [11]. Image segmentation is successfully accomplished by deep learning, however it is difficult to develop a measuring system for the extracted feature [15].

A close related work to ours is presented by [8]. Their work discusses an automated visual identification of individual great sharks from dorsal fin imagery. The approach addresses the dorsal fin as a textureless object, flexible and partially occluded. In order to identify biometricly an individual shark, they used a stroke model for fin detection, followed by a scale-space selective fingerprint to encode the fin individually. Also, they measure the species-specific distribution of visual uniqueness along the fin contour, and finally they propose a non-linear model for individual animal recognition and combine all approaches into a framework. This framework is classified as fine-grained since it takes into consideration the specific shape and the ridges of individual dorsal fin, multi-instanced since the images can be multi-labeled semantically according to the shark present, flexible since fins can bend, and fairly textureless for its lack of texture [8].

\section{Methodology}

\subsection{Boundary Descriptor}

Let $c(u)$ represent the contour of an object as following;

$$
c(u)=(x(u), y(u))
$$

where $u$ is the any parameter, and $x(u)$ and $y(u)$ denote coordinate functions of the contour, respectively. The contour is smoothed by convolution with the Gaussian function $G(u, \sigma)$, hence

$$
C(u, \sigma)=G(u, \sigma) * c(u)=(G(u, \sigma) * x(u), G(u, \sigma) * y(u))
$$


where $G(u, \sigma)=\frac{1}{\sqrt{2 \pi \sigma^{2}}} e^{-\frac{u^{2}}{2 \sigma^{2}}}, \sigma$ is a standard deviation, and $*$ denotes the convolution operator [25]. The corner response function is defined

$$
D(u, m, \sigma)=((G(u, m \sigma)-G(u, \sigma)) * x(u))^{2}+((G(u, m \sigma)-G(u, \sigma)) * y(u))^{2}
$$

where $m \sigma=m \times \sigma$, " $\times$ " denotes ordinary multiplication, i.e., $m>1$ is a multiplier factor [25]. Viewed as a bandpass filter, by varying $m$ and $\sigma$, the operator can be tuned to different frequency components of contour shape [8].

Definition 3.1. (Salient Locations) Let $S \prime$ be the set of salient locations defined by

$$
S \prime=\left\{u \mid \frac{\partial D(u, m, \sigma)}{\partial u}=0\right\}
$$

Definition 3.2. (Boundary Descriptor) Let $S$ "l be an ordered list of $u, \forall u \in S \prime$. For $u_{i} \in$ S'I where $i=1,2, \cdots,|S \prime \prime|-1$, we define the prominence function $p\left(u_{i}\right)=\mid D\left(u_{i}, m, \sigma\right)-$ $D\left(u_{i+1}, m, \sigma\right) \mid$. For $\delta$ largest values of $p\left(u_{i}\right)$ where $\delta$ is a user-defined parameter, we collect the respective $u_{i}$ 's in a multi-set $S$ called the boundary descriptor.

Definition 3.3. (Keypoints) The Keypoints of a contour is a subset of $c(u)$, which is denoted by $K$.

$$
K=\{c(u) \mid u \in S\}
$$

\subsection{Boundary Descriptor Distance}

Definition 3.4. (Critical Points) We define $c\left(u_{0}\right)=\left(x\left(u_{0}\right), y\left(u_{0}\right)\right)$ be the point at the back of the base of the fin, $c\left(u_{1}\right)=\left(x\left(u_{1}\right), y\left(u_{1}\right)\right)$ be the front of the base of the fin, and $\left(x\left(u_{2}\right), y\left(u_{2}\right)\right)$ be the point at the tip of the fin as shown in Figure 1.

We propose a transformation function to design an orientation and scale invariant distance between a pair of contours.

Definition 3.5. (Transformation Function) For a given contour $c(u)$ and its boundary descriptor $S$, the transformation function, $f: c(u)=(x(u), y(u)) \rightarrow C(u)=(X(u), Y(u))$ is defined by

$$
\left(\begin{array}{l}
X(u) \\
Y(u)
\end{array}\right)=\left(\begin{array}{cc}
\frac{1}{\alpha} & 0 \\
0 & \frac{1}{\alpha}
\end{array}\right)\left(\begin{array}{cc}
\cos \beta & \sin \beta \\
-\sin \beta & \cos \beta
\end{array}\right)\left(\begin{array}{l}
x^{\prime}(u) \\
y^{\prime}(u)
\end{array}\right)
$$

where $x^{\prime}(u)=x(u)-x\left(u_{0}\right), y^{\prime}(u)=y(u)-y\left(u_{0}\right), \alpha=\sqrt{\left(x\left(u_{2}\right)-x\left(u_{0}\right)\right)^{2}+\left(y\left(u_{2}\right)-y\left(u_{0}\right)\right)^{2}}$, and $\beta=\arctan \left(\frac{y\left(u_{2}\right)-y\left(u_{0}\right)}{x\left(u_{2}\right)-x\left(u_{0}\right)}\right)$.

Given two boundary descriptors $S_{1}$ and $S_{2}$, a bipartite graph $B\left(S_{1}, S_{2}\right)$ is defined with $E=\left\{(u, v) \mid \forall u \in S_{1}\right.$ and $\left.\forall v \in S_{2}\right\}$. For an edge $(u, v)$, the weight of the edge is defined by

$$
W(u, v)=\sqrt{(X(u)-X(v))^{2}+(Y(u)-Y(v))^{2}}
$$

where $(X(u), Y(u))=f((x(u), y(u)))$ and $(X(v), Y(v))=f((x(v), y(v)))$, which is the Euclidean distance between $(X(u), Y(u))$ and $(X(v), Y(v))$. 
Definition 3.6. (Boundary Descriptor Distance) The Boundary Descriptor Distance, $\mathcal{B D}\left(S_{1}, S_{2}\right)$, between two boundary descriptors $S_{1}$ and $S_{2}$ is the weight of the minimum weight perfect matching in $B\left(S_{1}, S_{2}, E\right)$ with the weighting scheme $W$.

Lemma 3.1. The boundary descriptor distance is $\mathcal{O}\left(n^{2.5} \log n\right)$ time computable where $n=$ $\left|S_{1}\right|=\left|S_{2}\right|$.

Proof. Let $S_{1}, S_{2}$ be boundary descriptors with $n$ elements. Computing the weights of the edges in $B\left(S_{1}, S_{2}\right)$ requires $\mathcal{O}\left(n^{2}\right)$ time. The minimum-weight perfect matching problem can be solved by using the faster scaling algorithm that is asymptotically bound by $\mathcal{O}\left(n^{2.5} \log (n)\right)$ [4], from which the desired runtime follows.

Lemma 3.2. The boundary descriptor distance is a metric, i.e., for boundary descriptors $S_{i}$, $S_{j}$, and $S_{k}$, the following four properties are satisfied.

1. $\mathcal{B D}\left(S_{i}, S_{j}\right) \geq 0$

(non-negativity)

2. $\mathcal{B D}\left(S_{i}, S_{j}\right)=0 \Longleftrightarrow S_{i}=S_{j}$

(identity)

3. $\mathcal{B D}\left(S_{i}, S_{j}\right)=\mathcal{B D}\left(S_{j}, S_{i}\right)$

(symmetry)

4. $\mathcal{B D}\left(S_{i}, S_{k}\right) \leq \mathcal{B D}\left(S_{i}, S_{j}\right)+\mathcal{B D}\left(S_{j}, S_{k}\right)$

(subadditivity)

Proof. Observe that non-negativity, identity, and symmetry properties follow directly from definition of the $\mathcal{B D}$ distance (i.e., Definition 3.6). To show the remaining subadditivity property suppose that $M_{i j}$ and $M_{j k}$ are the minimum weight perfect matchings in $B\left(S_{i}, S_{j}\right)$ and $B\left(S_{j}, S_{k}\right)$ respectively. Now, define a new matching in $B\left(S_{i}, S_{k}\right)$ by $M_{i k}:=\left\{(u, w) \mid(u, v) \in M_{i j} \wedge(v, w) \in\right.$ $\left.M_{j k}\right\}$, where $u \in S_{i}, v \in S_{j}$, and $w \in S_{k}$. For $u, v$, and $w, W(u, w) \leq W(u, v)+W(v, w)$ since the weighting function $W$ is based on the Euclidean distance which is a metric. We have

$$
\begin{array}{r}
\mathcal{B D}\left(S_{i}, S_{k}\right) \leq \sum_{(u, w) \in M_{i k}} W(u, w) \leq \sum_{(u, v) \in M_{i j},(v, w) \in M_{j k}}(W(u, v)+W(v, w)) \\
=\sum_{(u, v) \in M_{i j}} W(u, v)+\sum_{(v, w) \in M_{j k}} W(v, w)=\mathcal{B D}\left(S_{i}, S_{j}\right)+\mathcal{B D}\left(S_{j}, S_{k}\right)
\end{array}
$$

\section{Experimental Results}

We study the characteristics of the boundary descriptor distance by comparing mutated images of two shark fins. First, we captured the dorsal fin regions from two different sharks, named Shark 1 and Shark 2. Second, we augmented the number of dorsal fin images by applying the Affine Transformations which will be explained in the following section. Lastly, we demonstrate the distances of dorsal fin pairs of Shark 1-Shark 1 and Shark 1-Shark 2.

\subsection{Dataset}

Affine transformation preserves collinearity and ratio. For our work, we use the transformation to achieve the data augmentation process. Such transformations are expressed by matrix multiplications, and the usual way to represent them is a $2 \times 3$ matrix $M$. To transform a 2D-image of $S=\left[\begin{array}{ll}x y \\ y\end{array}\right]^{T}$ where $x$ and $y$ are the pixels of the original image, the transformed 


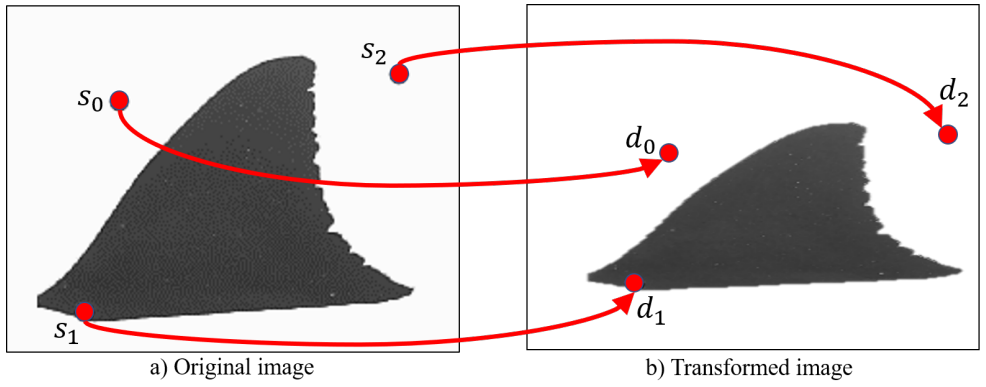

Figure 2: Example of the data augmentation created by applying Affine Transformations to the original image.

image is $T=M \cdot\left[\begin{array}{lll}x & y & 1\end{array}\right]^{T}$. Please refer [20] for further information. We compute the Affine transformation matrix $M$ for a 2-dimensional image given three points on the original image, $\left(s_{0}, s_{1}, s_{2}\right)$ and three points on the resulting image $\left(d_{0}, d_{1}, d_{2}\right)$. When $M$ is applied to the original image, the result is an image where $s_{i}$ corresponds to $d_{i}$ for $i \in\{0,1,2\}$. Figure 2 illustrates the process. We fix $\left(d_{0}, d_{1}, d_{2}\right)$ and iteratively generate $\left(s_{0}, s_{1}, s_{2}\right)$ with the function $s_{i}=\rho s_{i}^{\prime}+(1-\rho) r$ where $s_{i}^{\prime}$ is the previously generated point, $0 \leq \rho \leq 1$ is a momentum constant, and $r$ is a random number drawn from a uniform distribution.
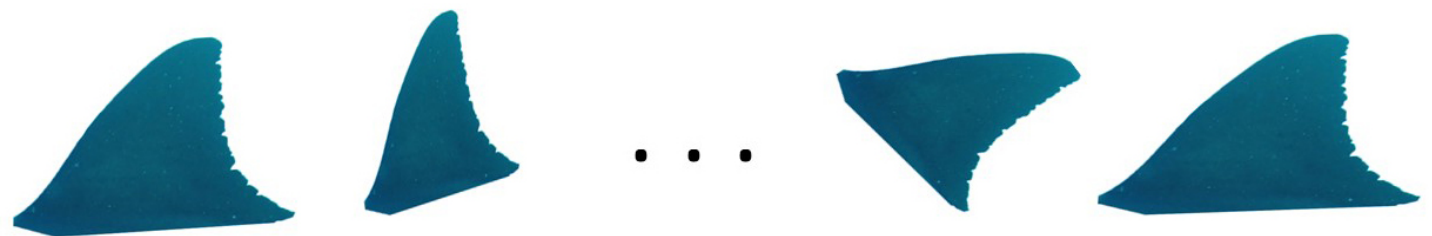

Figure 3: Samples of the mutated dorsal fin images.

We use images of two distinct sharks, Shark 1 and Shark 2, and we augmented the image data performing a series of Affine Transformations resulting in 250 dorsal fin images for both Shark 1 and Shark 2. Samples are shown in Figure 3. We then proceed to extract the contours by using Sobel edge detection and select 50 keypoints $(|K|=50)$ for each contour of a dorsal fin image. We define $S_{i, k}$ to be the boundary descriptor of $i^{\text {th }}$ dorsal fin image of the Shark $k$ where $i \in\{1, \cdots, 250\}$ and $k \in\{1,2\}$.

\subsection{Distribution of pairwise distances from two sharks}

We compute the boundary descriptor distance $\mathcal{B D}\left(S_{1,1}, S_{i, 1}\right), \forall i \in\{2, \cdots, 250\}$, and their distributions are shown in Figure 5 with the legend Shark 1 - Shark 1. Similarly, we compute the boundary descriptor distance $\mathcal{B D}\left(S_{1,1}, S_{j, 2}\right), \forall j \in\{1, \cdots, 250\}$, and their distributions are shown with the legend Shark 1 - Shark 2. In addition, the descriptive statistics are summarized in Table 1.

Even it is clear that two distributions are distinguished, we performed the Wilcoxon signedrank test between two distributions to verify they are not identical shark, and it's $p$-value $<2.2 e^{-16}$, which accepts our hypothesis. 


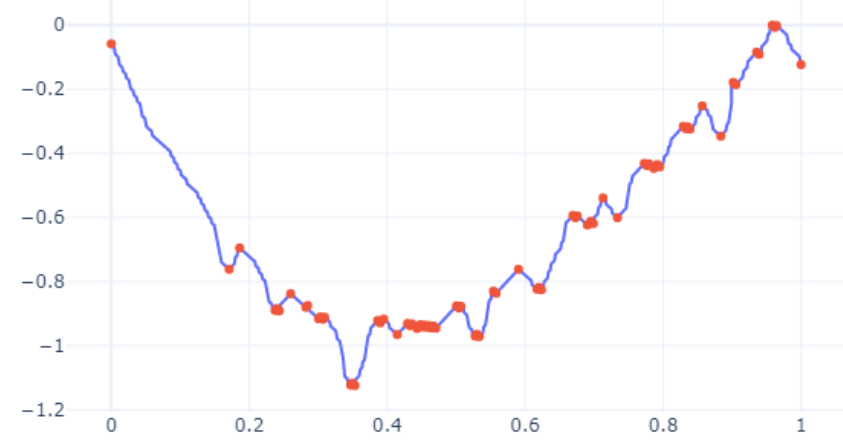

Figure 4: Example of keypoints extraction for Shark 1.

\section{Distribution of boundary descriptor distances \\ Pair $\square$ Shark 1 - Shark $1 \square$ Shark 1 - Shark 2}

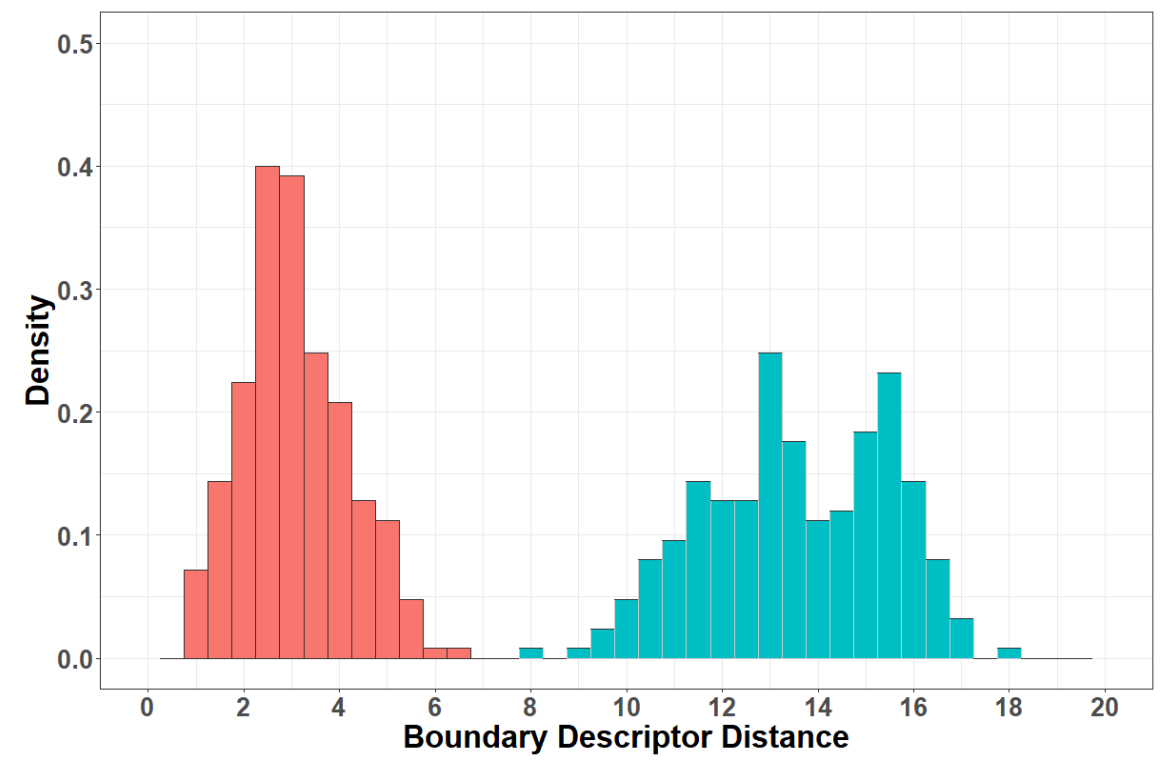

Figure 5: Distribution of the boundary descriptor distances between randomly mutated dorsal fin images from two different sharks.

\section{Conclusion}

In this paper, we described a metric for boundary descriptors based on bipartite perfect matching applied in shark dorsal fins that later will be used in a framework to identify and track individuals from visual data. To do so, we proposed a method to find the keypoints of a contour and a transformation function to perform a minimum weight perfect matching in a bipartite graph. Then, we could show that our metric is applicable to individual shark identification. 
Table 1: Descriptive statistics of the the boundary descriptor distances between randomly mutated dorsal fin images from two different sharks. (SD: standard deviation)

\begin{tabular}{c||c|c}
\multicolumn{1}{c||}{} & \multicolumn{2}{c}{ Boundary Descriptor Distance } \\
\hline \hline & Shark 1- Shark 1 & Shark 1 - Shark 2 \\
\hline Mean & 3.08 & 13.56 \\
SD & 1.10 & 1.91 \\
Median & 2.93 & 13.53 \\
Min & 0.86 & 8.09 \\
Max & 6.29 & 18.03
\end{tabular}

\section{References}

[1] Ali Ismail Awad. From classical methods to animal biometrics: A review on cattle identification and tracking. Computers and Electronics in Agriculture, 123:423-435, 2016.

[2] Anthony I Dell, John A Bender, Kristin Branson, Iain D Couzin, Gonzalo G de Polavieja, Lucas PJJ Noldus, Alfonso Pérez-Escudero, Pietro Perona, Andrew D Straw, Martin Wikelski, et al. Automated image-based tracking and its application in ecology. Trends in ecology $\&$ evolution, 29(7):417-428, 2014.

[3] Michael L Domeier and Nicole Nasby-Lucas. Annual re-sightings of photographically identified white sharks (carcharodon carcharias) at an eastern pacific aggregation site (guadalupe island, mexico). Marine Biology, 150(5):977-984, 2007.

[4] Jack Edmonds and Richard M Karp. Theoretical improvements in algorithmic efficiency for network flow problems. Journal of the ACM (JACM), 19(2):248-264, 1972.

[5] Jan Flusser and Tomas Suk. Pattern recognition by affine moment invariants. Pattern Recognition, 26(1):167-174, 1993.

[6] Kevin J Gaston and Mark A O'Neill. Automated species identification: why not? Philosophical Transactions of the Royal Society of London B: Biological Sciences, 359(1444):655-667, 2004.

[7] Lex Hiby, Phil Lovell, Narendra Patil, N Samba Kumar, Arjun M Gopalaswamy, and K Ullas Karanth. A tiger cannot change its stripes: using a three-dimensional model to match images of living tigers and tiger skins. Biology Letters, pages rsbl-2009, 2009.

[8] Benjamin Hughes and Tilo Burghardt. Automated visual fin identification of individual great white sharks. International Journal of Computer Vision, 122(3):542-557, 2017.

[9] Marcella J Kelly. Computer-aided photograph matching in studies using individual identification: an example from serengeti cheetahs. Journal of Mammalogy, 82(2):440-449, 2001.

[10] Hjalmar S Kühl and Tilo Burghardt. Animal biometrics: quantifying and detecting phenotypic appearance. Trends in ecology $E$ evolution, 28(7):432-441, 2013.

[11] Santosh Kumar, Amit Pandey, K Sai Ram Satwik, Sunil Kumar, Sanjay Kumar Singh, Amit Kumar Singh, and Anand Mohan. Deep learning framework for recognition of cattle using muzzle point image pattern. Measurement, 116:1-17, 2018.

[12] Santosh Kumar and Sanjay Kumar Singh. Visual animal biometrics: survey. IET Biometrics, 6(3):139-156, 2016.

[13] Mayank Lahiri, Chayant Tantipathananandh, Rosemary Warungu, Daniel I Rubenstein, and Tanya Y Berger-Wolf. Biometric animal databases from field photographs: identification of individual zebra in the wild. In Proceedings of the 1st ACM international conference on multimedia retrieval, page 6. ACM, 2011.

[14] Yu Lin, Vaibhav Rajan, and Bernard ME Moret. A metric for phylogenetic trees based on matching. IEEE/ACM Transactions on Computational Biology and Bioinformatics (TCBB), 9(4):1014- 
$1022,2012$.

[15] Jonathan Long, Evan Shelhamer, and Trevor Darrell. Fully convolutional networks for semantic segmentation. In Proceedings of the IEEE conference on computer vision and pattern recognition, pages 3431-3440, 2015.

[16] AD Marshall and SJ Pierce. The use and abuse of photographic identification in sharks and rays. Journal of Fish Biology, 80(5):1361-1379, 2012.

[17] Jucheol Moon and Oliver Eulenstein. Cluster matching distance for rooted phylogenetic trees. In International Symposium on Bioinformatics Research and Applications, 2018.

[18] Elena Ranguelova, Mark Huiskes, and Eric J Pauwels. Towards computer-assisted photoidentification of humpback whales. In Image Processing, 2004. ICIP'04. 2004 International Conference on, volume 3, pages 1727-1730. IEEE, 2004.

[19] Wim Rossing. Animal identification: introduction and history. Computers and Electronics in Agriculture, 24(1-2):1-4, 1999.

[20] Philip Schneider and David H Eberly. Geometric tools for computer graphics. Elsevier, 2002.

[21] Conrad W Speed, Mark G Meekan, and Corey JA Bradshaw. Spot the match-wildlife photoidentification using information theory. Frontiers in Zoology, 4(1):2, 2007.

[22] R Stanley. Darwin: Identifying dolphins from dorsal fin images. Senior Thesis, Eckerd College, 1995.

[23] Alison V Towner, Michelle A Wcisel, Ryan R Reisinger, David Edwards, and Oliver JD Jewell. Gauging the threat: the first population estimate for white sharks in south africa using photo identification and automated software. PLOS ONE, 8(6):e66035, 2013.

[24] AM Van Tienhoven, JE Den Hartog, RA Reijns, and VM Peddemors. A computer-aided program for pattern-matching of natural marks on the spotted raggedtooth shark carcharias taurus. Journal of Applied Ecology, 44(2):273-280, 2007.

[25] Xiaohong Zhang, Honxing Wang, Mingjian Hong, Ling Xu, Dan Yang, and Brian C Lovell. Robust image corner detection based on scale evolution difference of planar curves. Pattern Recognition Letters, 30(4):449-455, 2009. 